\title{
Study of the effect of shell closure on the nuclear dissipation
}

\author{
V. Singh ${ }^{1}$, B.R. Behera ${ }^{1 *}$, M. Kaur ${ }^{1}$, D. Siwal' ${ }^{2}$, S. Goyal ${ }^{2}$, P. Sugathan ${ }^{3}$, K.S. Golda ${ }^{3}$, A. Jhingan ${ }^{3}$, \\ A. Kumar ${ }^{1}$, A. Saxena ${ }^{4}$, R.K. Bhowmik ${ }^{3}$ and S. Kailas ${ }^{4}$ \\ ${ }^{1}$ Department of Physics, Panjab University, Chandigarh-160014, India \\ ${ }^{2}$ Department of Physics and Astrophysics, Delhi University, Delhi-110007, India \\ ${ }^{3}$ Inter University Accelerator Centre, Aruna Asaf Ali Marg, New Delhi-110067, India \\ ${ }^{4}$ Nuclear Physics Division, Bhabha Atomic Research Centre, Mumbai- 499985, India \\ *E-Mail:- bivash@pu.ac.in
}

\begin{abstract}
Neutron multiplicity measurements are carried out for the ${ }^{19} \mathrm{~F}+{ }^{194,196,198} \mathrm{Pt}$ systems at excitation energy range of 48 to $67.5 \mathrm{MeV}$. Out of these three compound nuclei $(\mathrm{CN}){ }^{217} \mathrm{Fr},{ }^{215} \mathrm{Fr}$ and ${ }^{213} \mathrm{Fr}$, one of the $\mathrm{CN}$ $\left({ }^{213} \mathrm{Fr}\right)$ has neutron shell closure $\left(\mathrm{N}_{\mathrm{C}}=126\right)$. Experimentally obtained neutron multiplicities are compared with the standard statistical model predictions. It has been observed that the standard statistical model under-predicts the experimental pre-scission neutron multiplicities values. Also the comparison of pre-scission neutron multiplicities for different compound nuclei $(\mathrm{CN})$ indicates the signature of shell effects on nuclear dissipation, which results in the lowering of the pre-scission neutron multiplicities.
\end{abstract}

\section{Introduction}

In the last century the development of nuclear accelerator for the production of heavy ion beams opened up an opportunity to study heavy-ion induced fusion-fission reactions. The importance of nuclear dissipation in the fusion-fission dynamics is well established from the study of the light charged particles, neutrons and giant dipole resonance (GDR) gamma rays emission during heavy-ion induced fusion-fission reaction [1]. Experimental signatures of large dissipation is observed through the observation of excess pre-fission neutrons, gamma ray multiplicities from the compound nucleus giant dipole resonance (GDR), light charged particles and the evaporation residue [2]. Mainly, dissipation is observed at nuclear temperature between 1 and $2 \mathrm{MeV}$, also it is found that, dissipation effect increases with the increase in excitation energy.

On the other hand, production of new super-heavy elements is one of the contemporary goals of nuclear physics. Attempts are being made to explore the predicted island of super-heavy elements (SHE) and to find what will be the next proton and neutron magic nucleus beyond 82 and 126 respectively. Different theoretical models predict proton and neutron shell closure at $\mathrm{Z}=114,120,126$ and $\mathrm{N}=172,184$ [3, 4]. In order to achieve these goals, extensive knowledge of fusion-fission dynamics of heavy nuclei is essential.
The effect of the shell closure on nuclear dissipation can be observed by a number of probes (like evaporation residue cross-sections, fission cross-sections, neutron multiplicity, charged particle multiplicity etc). Back et al. [5] have measured the Evaporation residue (ER) crosssections for the ${ }^{216} \mathrm{Th}$ nuclei and reported the effect of the shell closure on nuclear dissipation. But the conclusion drawn from more than one observable sensitive to nuclear dissipation will give a more accurate behaviour of nuclear dissipation against shell effect. To explore this effect, we have planned experiments to measure ER cross-section, fission cross-section and neutron multiplicity for the ${ }^{19} \mathrm{~F}+$ ${ }^{194,196,198} \mathrm{Pt}$ systems, and we also plan to perform a simultaneous analysis of these observables. The compound nuclei formed in these cases are with the neutron numbers varying from 126 to 130 . Here we report the results of the neutron multiplicity measurements for the above mentioned systems.

The experimental set-up is described in the next section. Section 3 contains the procedure for extracting the neutron multiplicities from the experimental data. Details of the statistical model calculations are given in the section 4 . The conclusion of the work is given in the last section.

\section{Experimental set up}

The experiment was performed using 15UD pelletron accelerator and National Array of Neutron Detectors 
(NAND) facility at IUAC, New Delhi. Pulsed beam of ${ }^{19} \mathrm{~F}$ (Energy Range $=90-115 \mathrm{MeV}$ ) at a repetition rate of 250 ns and pulse width of 1.5 ns, was bombarded on the targets of ${ }^{194} \mathrm{Pt},{ }^{196} \mathrm{Pt}$ and ${ }^{198} \mathrm{Pt}$ of thickness 1.75 $\mathrm{mg} / \mathrm{cm}^{2}, 1.8 \mathrm{mg} / \mathrm{cm}^{2}$ and $2.15 \mathrm{mg} / \mathrm{cm}^{2}$ respectively. The targets were fabricated by rolling technique. These targets were located at the centre of a thin walled spherical scattering chamber of $60 \mathrm{~cm}$ diameter. Fission fragments were detected by a pair of Multi-wire proportional counter (MWPC) (Active area $=5 ” \times 3$ ” ) kept at the fission fragment folding angle at a distance of $18.5 \mathrm{~cm}$ and $17.0 \mathrm{~cm}$ from the target position. The MWPC were operated at 3.5 torr pressure of iso-butane gas and $-430 \mathrm{~V}$ bias. A clean separation between fission-fragments and elastically scattered beam was obtained.

Neutrons were detected by 16 NE213 organic scintillator (5" dia $\times 5$ " thick) coupled to a Photonics (XP4512B) photomultiplier tube. Out of these 16 detectors, 12 detectors were kept at a distance of 2 meter and the remaining 4 detectors were kept at 1 meter from the target. These detectors were placed at different angles ranging from $30^{\circ}$ to $315^{\circ}$ around the target chamber in reaction plane as shown in Fig. 1. A hardware threshold of $0.5 \mathrm{MeV}$ of neutron energy was applied on neutron detectors using ${ }^{137} \mathrm{Cs}$ and ${ }^{60} \mathrm{Co}$ sources [6]. Two silicon surface barrier detectors were also placed inside the chamber at azimuthal angle $\pm 16^{\circ}$ to the beam direction out of the reaction plane for the normalization purpose.

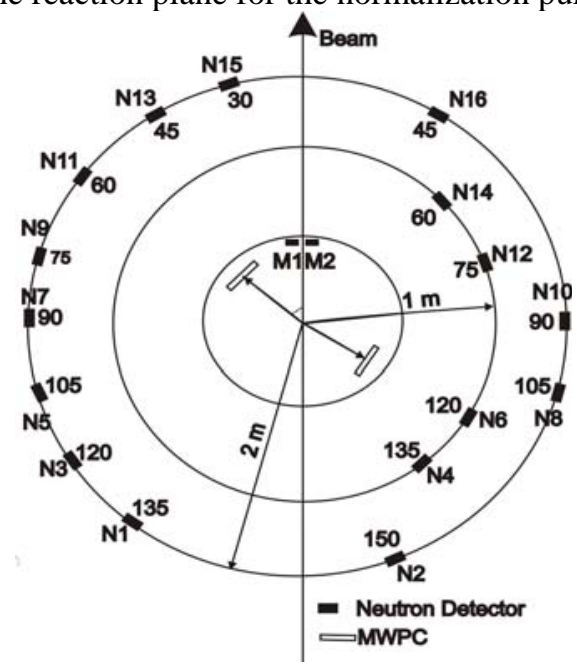

Fig. 1. Schematic diagram of the neutron detector array.

The trigger for the data acquisition was generated by the Logical OR of cathode signals of the two MWPC further ANDed with the RF of the beam pulse. Neutron gamma discrimination was performed by using both time of flight (TOF) and pulse shape discrimination module (PSD) based on the zero cross-over techniques [7]. In order to keep the background at a minimum level, the beam dump was kept at a distance of $3 \mathrm{~m}$ from the target position and was shielded with paraffin blocks and lead bricks. Data acquisition was carried out using CAMAC based software CANDLE [8].

\section{Data analysis}

Neutrons detected in coincidence with the fission fragments are assumed to originate from three moving sources (compound nucleus evaporation and two fission fragments). The compound nucleus contribution (prescission) and contribution from fission fragments (postscission) are assumed to be isotropic. Further, postscission neutron multiplicity and temperatures are assumed to be same for both the fragments. Hence the total neutron multiplicity $\mathrm{M}_{\text {total }}=\mathrm{M}_{\text {pre }}+2 * \mathrm{M}_{\text {post }}$. The contribution for each source of neutrons was obtained by multiple moving sources fitting using least square method. The raw neutron TOF spectra have been calibrated using gamma peak as reference. 2 dimensional PSD gate have been applied to separate neutron and gamma. Calibrated and PSD gated spectra have been converted to energy spectra for all 16 in plane detectors. Energy spectra obtained experimentally was corrected with the efficiency, obtained with a Monte-carlo efficiency code MODEFF and also with the measured experimental efficiency using a ${ }^{252} \mathrm{Cf}$ source kept at the target position. In order to obtain pre-scission and postscission contributions spectra of 16 detectors were fitted simultaneously for 32 different neutron-fission angle ( $\Phi_{\mathrm{nf}}$ ) combinations, with watt expression given by:

$Y\left(E_{n}\right)=\sum_{i=1}^{3} \frac{M_{n}^{i} \sqrt{E_{n}}}{2\left(\pi T_{i}\right)^{3 / 2}} \times \exp \left[\frac{-\left(E_{n}-2 \sqrt{\varepsilon_{i} E_{n}} \operatorname{Cos} \Phi_{i}+\varepsilon_{i}\right)}{T_{i}}\right]$

Where $\varepsilon_{i}, T_{i}$ and $M_{n}^{i}$ are energy per nucleon, temperature and multiplicity of neutron source $i$. $E_{n}$ is lab energy of neutrons and $\phi_{i}$ is neutron detection angle with respect to source $i$.

Energy of fission fragment was obtained using Viola systematics [9] for symmetric fission. The neutron multiplicities obtained by using $\mathrm{M}_{\text {pree }}, \mathrm{M}_{\text {post }}, \mathrm{T}_{\text {pre }}$, and $\mathrm{T}_{\text {post }}$ as free parameters. Neutron multiplicities obtained from fitting for decay of ${ }^{213} \mathrm{Fr},{ }^{215} \mathrm{Fr}$ and ${ }^{217} \mathrm{Fr}$ at different excitation energies are given in Fig. 2 and the three moving source fitting plots for few detectors are shown in the Fig. 3.

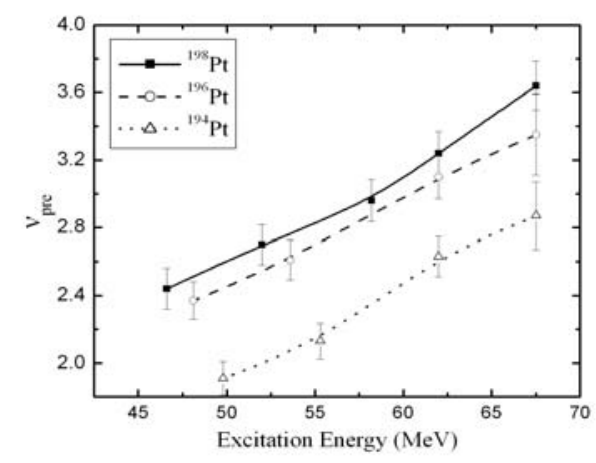

Fig. 2. Variation of $v_{\text {pre }}$ with excitation energy for the different systems. 


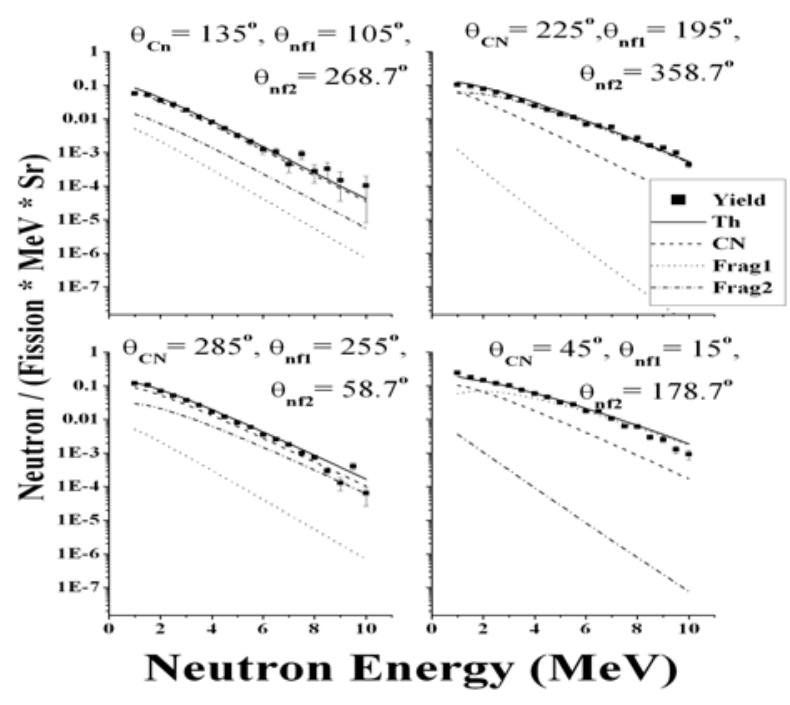

Fig. 3. Neutron multiplicity (filled circle) for the ${ }^{19} \mathrm{~F}+{ }^{198} \mathrm{Pt}$ system at $\mathrm{E}_{\mathrm{ex}}=52.0 \mathrm{MeV}$ along with the fits for the pre-scission (dot curve) and post-scission from one fragment (dot dashed curve) and that from the other (dot dot dash curve). The solid curve represents the total contribution.

\section{Statistical model calculations}

Total neutron multiplicity obtained for all the systems matches with the systematic given by Hinde et al. [10] for a wide range of systems with various fissility and excitation energies. Also the values of pre-scission neutron multiplicity are in agreement with the Baba systematic [11]. Statistical model calculation (PACE2) has been performed using different set of statistical model parameters. It is observed that pre-scission neutron multiplicity depends strongly on the ratio of level density at saddle to the level density at equilibrium $\left(a_{\mathrm{f}} / \mathrm{a}_{\mathrm{n}}\right)$ as shown in Fig. 4.

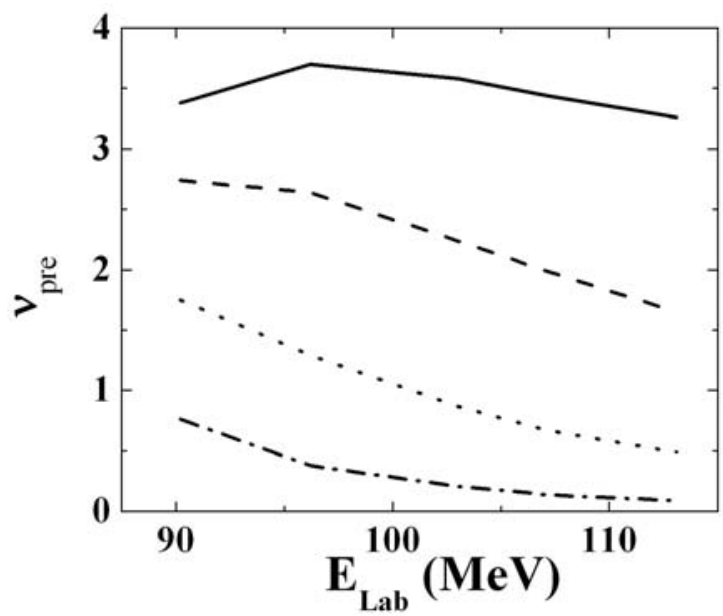

Fig. 4. Neutron multiplicities obtained from the statistical model code (PACE2) using different values of $a_{f} / a_{n}$. Here $a_{f} / a_{n}=$ 1.00 (solid line), $\mathrm{a}_{\mathrm{f}} / \mathrm{a}_{\mathrm{n}}=1.05$ (dash line), $\mathrm{a}_{\mathrm{f}} / \mathrm{a}_{\mathrm{n}}=1.10$ (dot line), $\mathrm{a}_{\mathrm{f}} / \mathrm{a}_{\mathrm{n}}=1.15$ (dash dot line).
Whereas the fission barrier paramter $\left(\mathrm{k}_{\mathrm{f}}\right)$ does not affect the multiplicity values much. Hence, an appropriate set of statistical model parameter should be used for the calculations. The value of $\mathrm{a}_{\mathrm{f}} / \mathrm{a}_{\mathrm{n}}=1.015, \mathrm{k}_{\mathrm{f}}=1.17$ for ${ }^{19} \mathrm{~F}$ $+{ }^{194} \mathrm{Pt}$ system and $\mathrm{a}_{\mathrm{f}} / \mathrm{a}_{\mathrm{n}}=1.050, \mathrm{k}_{\mathrm{f}}=1.17$ for ${ }^{19} \mathrm{~F}+{ }^{198} \mathrm{Pt}$ system are used to perform the statistical model calculation. These parameters are obtained by fitting the measured fission and ER cross sections for ${ }^{19} \mathrm{~F}+{ }^{194} \mathrm{Pt}$ and ${ }^{19} \mathrm{~F}+{ }^{198} \mathrm{Pt}$ systems [12]. For ${ }^{19} \mathrm{~F}+{ }^{196} \mathrm{Pt}$ system fission and ER cross-section data are not available, hence for performing the calculations, the same parameters are used, as in ${ }^{19} \mathrm{~F}+{ }^{194} \mathrm{Pt}$ system. A comparison of the experimental data and PACE2 predictions for different systems are shown in the Fig. 5.

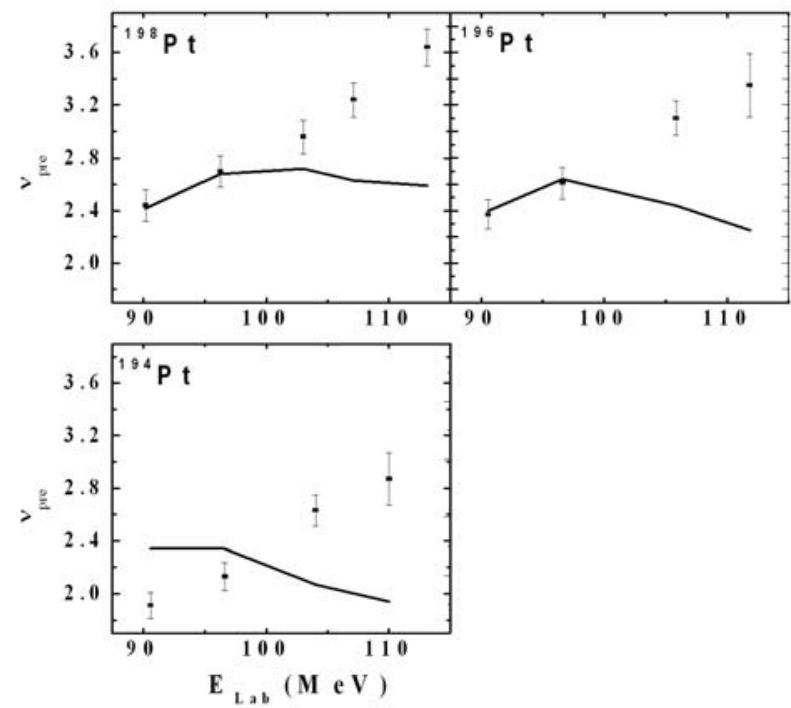

Fig. 5. Comparison of experimentally obtained pre-scission neutron multiplicities (filled square) with statistical model PACE2 (Solid line) prediction.

At the lowest excitation energy PACE2 predictions could reproduce the experimental results for pre-scission neutron multiplicity for ${ }^{19} \mathrm{~F}+{ }^{198} \mathrm{Pt}$ and ${ }^{19} \mathrm{~F}+{ }^{196} \mathrm{Pt}$ systems. However, at higher excitation energy the measured neutron multiplicity values deviate considerably from the predictions. This may be precisely due to the fact that, nuclear dissipation effect is not included in the PACE2 calculation.

\section{Summary and conclusions}

We have measured the neutron multiplicities for different isotopes of $\mathrm{Fr}$ at various excitation energies. It is interesting to compare the neutron multiplicities for different systems at same excitation energies (see Fig. 2). As we go from ${ }^{194} \mathrm{Pt}$ to ${ }^{198} \mathrm{Pt}$ through ${ }^{196} \mathrm{Pt}$ target, there is a gradual increase of two neutrons. However, the measured multiplicity variation of ${ }^{194} \mathrm{Pt}$ to ${ }^{196} \mathrm{Pt}$ target is more as compared to the values for the ${ }^{196} \mathrm{Pt}$ to ${ }^{198} \mathrm{Pt}$ target. This discrepancy may be attributed to the shell closure of $\mathrm{N}_{\mathrm{C}}=$ 126 in ${ }^{19} \mathrm{~F}+{ }^{194} \mathrm{Pt}$ system. The experimentally obtained pre-scission neutron multiplicity values are compared with the statistical model calculations. It has been observed that statistical model under-predicts the experimentally obtained pre-scission neutron multiplicities at most of the energies. However, the 
deviation for the system ${ }^{19} \mathrm{~F}+{ }^{194} \mathrm{Pt}$, at higher excitation energies is less compared to other two systems. It may be due to the presence of neutron shell closure $\left(\mathrm{N}_{\mathrm{c}}=126\right)$ in the former system. This also indicates that the threshold for dissipative effects is higher for ${ }^{19} \mathrm{~F}+{ }^{194} \mathrm{Pt}$ system which is having $\mathrm{N}_{\mathrm{c}}=126$. Back et al. [5] has indicated that the systems with $\mathrm{N}_{\mathrm{c}}=126$ may have higher excitation energy threshold for nuclear dissipation effects. A consistent analysis of the data is in progress to determine these effects.

\section{Acknowledgment}

The financial support from the Council of Scientific \& Industrial Research (CSIR), Govt. of India, in terms of a SPM Fellowship to Varinderjit Singh is gratefully acknowledged. BRB acknowledge the Department of Atomic Energy (DAE), Govt. of India for the DAE Young Scientist Research Award. A special thanks to Dr. A. Shirvastava for presenting our work at Fusion 2011 conference.

\section{References}

[1] D. Hilscher and H. Rossner, Ann. Phys. Fr. 17, 471 (1992).

[2] Peter Paul, Michael Thoennessen Annu. Rev. Nucl. Part. Sci 44, 65-108 (1994).

[3] K. Rutz, M. Bender, T. B"urvenich, T. Schilling, P.G. Reinhardt, J. A. Maruhn and W. Greiner, Phys. Rev. C 56, 238 (1997).

[4] R. K. Gupta, M. Balasubramaniam, C. Mazzocchi, M. La. Commara and W. Scheid, Phys. Rev. C 65, 024601 (2002).

[5] B. B. Back, D. J. Blumenthal, C. N. Davids, D. J. Henderson, R. Hermann, D. J.Hofman, C. L. Jiang, H. T. Penttila and A. H. Wuosmaa, Phys. Rev. C 60, 044602 (1999).

[6] T. G. Masterson, Nucl. Instrum. Methods 88, 61 (1970).

[7] S. Venkataramanan, Arti Gupta, K.S. Golda, Hardev Singh, Rakesh Kumar, R.P. Singh and R.K. Bhowmik, Nucl. Instr. and Meth. A 596, 248 (2008).

[8] E. T. Subramaniam, Kusum Rani, B. P. Ajith Kumar, and R. K. Bhowmik, Rev. Sci. Instr. 77, 096102 (2006).

[9] V.E. Viola, K. Kwiatkowski and M. Walker, Phys. Rev. C 31, 1550 (1985).

[10] D. Hinde et. al. Nucl. Phys. A 452, 550 (1986).

[11] Hiroshi Baba, Atsushi Shinohara, Tadashi Saito, Naruto Takahashi and Akihiko Yokoyama, Journal of Physical Society of Japan 66, No. 4, 998 (1997).

[12] K.Mahata, S. Kailas, A. Shirvastava, A. Chatterjee, P. Singh, S. Santra and B. S.Tomar Phys. Rev. C 65, 034613 (2002). 